\title{
Multiplier systems
}

\author{
by \\ Paul C. Pasles (Villanova, PA)
}

1. Introduction. A multiplier system is a factor associated with a modular form. Even more generally, it can be defined without recourse to an underlying form.

A multiplier system on a subgroup of $\mathrm{SL}(2, \mathbb{R})$ is a concept analogous to that of a group character, but with a slightly more complicated consistency condition which includes one or two extra parameters (weights). Because of their importance in modular forms theory, these objects were studied by Hecke, Rademacher \& Zuckerman, and Petersson, among others [8, 28, $30,31]$. The multiplier systems possible for complex weight are considered in the present paper. We also present an upper bound on the number of multipliers possible for a given group and weight. Finally, we apply our approach to extend a convergence result in the theory of modular forms.

Of particular interest to us is the Hecke group $\left.G_{\lambda}=\left\langle S_{\lambda}, T\right\rangle, \lambda\right\rangle 0$, where $S_{\lambda}=\left(\begin{array}{ll}1 & \lambda \\ 0 & 1\end{array}\right)$ and $T=\left(\begin{array}{cc}0 & -1 \\ 1 & 0\end{array}\right)$.

Multipliers of a single real weight have been characterized for the modular group $G_{1}$ (see [31]), for two other Hecke groups [11,33] and for discrete (and some nondiscrete) Hecke groups [6,7]. We will obtain like results for the more general scenario: two "coweights" instead of one weight; neither coweight necessarily real; no technical assumptions regarding $v(-I)$; no bound on the multiplier system $v$. The last of these is motivated by the fact that the traditional restriction $|v| \equiv 1$ has been relaxed in some recent applications [15, 24-26].

To see that our very general definition is a meaningful one requires a demonstration that such multiplier systems exist for any given coweights. This was shown for the theta group in [24], but since the focus of the current paper will be triangle groups, we present a construction for one of the latter.

The important role of multiplier systems in analytic number theory is well established, with a close connection - via modular forms - to arithmetic

2000 Mathematics Subject Classification: Primary 11F11; Secondary 11F37.

Key words and phrases: multiplier system, Hecke group, modular form, Poincaré series. 
functions such as the partition function $p(n)$ and the sums-of-squares enumerator $r_{s}(n)$ (see $[14,2]$ ). Several recent papers have also examined modular and automorphic forms and integrals of nonreal weight [24-26]; their allied multipliers are among those to be considered in this paper. In particular, we will describe a relation between four parameters associated with multipliers: $\alpha$ (often called the weight or first coweight), $\beta$ (the coweight or second coweight), $\lambda$ (the width), and $\kappa=1 / 2+(2 \pi i)^{-1} \log \left[-v\left(S_{\lambda}\right)\right]$, all of which will be carefully defined in due course.

Forms exist on the modular group for any complex weight (or pair of coweights), as exemplified by those we will construct in our first proof. Nonetheless, Poincaré series on that group diverge absolutely if the coweight difference is not real, so the usual method of basis construction will not work in that situation. The current author proved this divergence result [25] except in the case $\left|v\left(S_{1}\right)\right| \neq 1$; we will prove that the extra assumption is unnecessary, and in fact that $\left|v\left(S_{1}\right)\right|=1$ if and only if the coweight difference is real.

2. Multiplier systems. We begin with a precise definition of the central concept. We will take a quite general view, essentially in accordance with $[24,25]$, to obtain a result with broad applicability.

Following [20], take $\arg (c \bar{z}+d) \in(-\pi, \pi]$ for $\operatorname{Im} z>0$ and $c, d \in \mathbb{R}(c$ and $d$ not both zero), and $\arg w \in[-\pi, \pi)$ otherwise. This ensures usefully that $\arg (c z+d)=-\arg (c \bar{z}+d)$ and $\log (c z+d)+\log (c \bar{z}+d) \in \mathbb{R}$, thus simplifying many calculations. As usual define logarithms by $\log w:=\log |w|+i \arg w$ and exponentiation by $w^{r}=\exp \{r \log w\}$.

Definition. If $G$ is a group of real $2 \times 2$ matrices and $\alpha, \beta \in \mathbb{C}$ then $v: G \rightarrow \mathbb{C} \backslash\{0\}$ is called a multiplier system of coweights $\alpha, \beta$ on $G$ iff

$$
\begin{aligned}
& v\left(M_{3}\right)\left(c_{3} z+d_{3}\right)^{\alpha}\left(c_{3} \bar{z}+d_{3}\right)^{\beta} \\
& \quad=v\left(M_{1}\right)\left(c_{1} M_{2} z+d_{1}\right)^{\alpha}\left(c_{1} M_{2} \bar{z}+d_{1}\right)^{\beta} v\left(M_{2}\right)\left(c_{2} z+d_{2}\right)^{\alpha}\left(c_{2} \bar{z}+d_{2}\right)^{\beta},
\end{aligned}
$$

whenever $M_{j}=\left(\begin{array}{ll}a_{j} & b_{j} \\ c_{j} & d_{j}\end{array}\right), M_{3}=M_{1} M_{2}$, for $c, d \in \mathbb{R}^{2} \backslash\{(0,0)\}$ and $z \in \mathcal{H}=$ the upper half-plane $\{z: \operatorname{Im} z>0\}$. (The above equation is known as the consistency condition.)

When $\alpha-\beta \in \mathbb{Z}$, the consistency condition reduces to multiplicativity: $v$ is then a homomorphism.

Note. Although a two-coweight approach is not necessary in the multiplier system point of view, it makes quite a difference in the immediate application, namely automorphic forms and integrals. A multiplier of coweights 3,0 automatically serves as a multiplier of coweights 5,2 , it is true; but the same cannot be said of a form of coweights 3,0 . Thus the two-coweight definition is meaningful. 
Several textbooks treat multiplier systems of real weight, for example the now classic $[14,19]$ and the more recent [9]. As examples, we have the multiplier systems associated with modular forms such as the classical eta and theta functions. The reader is referred to various derivations of the eta multiplier in $[30,28,14,2]$ and the theta multiplier in [14].

Ordinarily one includes further restrictions in the definition, such as that $\alpha \in \mathbb{R}, \beta=0$, and $|v| \equiv 1$ (e.g. [6, 21, 13, 12]), or simply that $\left|v\left(S_{\lambda}\right)\right|=1$ (as in [25]), or even that $v\left(S_{\lambda}\right)=1$ (see $\left.[24,26]\right)$. A few authors further restrict to $\alpha \in \mathbb{Z}, \beta=0$, and $v \equiv 1$ (e.g. [3, 32]). Our definition requires none of these assumptions, and to justify it we will give a relevant construction for arbitrary $\alpha, \beta$.

Thus, our results are quite general in that they allow two coweights, neither necessarily real, and no restriction on $v(-I)$ nor on $|v| ; v$ is not bounded. In each of these ways our definition is more general than those presented elsewhere. On the other hand, our results are specific to the Hecke groups, not to other groups whose multiplier systems have been studied $[1,5,17,18,29,34]$.

Our first proposition establishes existence of multiplier systems of any arbitrary complex coweights for one important group. Simultaneously, the proof indicates why we do not restrict to $|v| \equiv 1$.

Proposition 1 (Existence of multipliers). For any $\alpha, \beta \in \mathbb{C}$, there exists a multiplier system of coweights $\alpha, \beta$ on the modular group $G_{1}=$ $\left\langle\left(\begin{array}{ll}1 & 1 \\ 0 & 1\end{array}\right),\left(\begin{array}{cc}0 & -1 \\ 1 & 0\end{array}\right)\right\rangle$. (It follows immediately that multiplier systems exist on the infinitely many Hecke groups $G_{n}, n \in \mathbb{Z}^{+}$.)

Proof. Put $\alpha_{0}=\alpha+2|\lfloor\operatorname{Re} \alpha\rfloor|, \beta_{0}=\beta+2|\lfloor\operatorname{Re} \beta\rfloor|$ and let $\Delta$ denote the discriminant function $\Delta(z):=(2 \pi)^{12} e^{2 \pi i z} \prod_{n=1}^{\infty}\left(1-e^{2 \pi i n z}\right)^{24}$, where $\operatorname{Im} z>0$. In our terminology, $\Delta$ is a modular form of coweights 12,0 :

$$
\Delta(-1 / z)=z^{12} \Delta(z), \quad \operatorname{Im} z>0 .
$$

Observe that $\operatorname{Re} \alpha_{0}, \operatorname{Re} \beta_{0}>0$. It can be proved that for $\operatorname{Re} \gamma>0$, we have $\Delta^{\gamma}(-1 / z)=e^{-6 \pi i \gamma} z^{12 \gamma} \Delta^{\gamma}(z)$ and $\Delta^{\gamma}(1+z)=e^{2 \pi i \gamma} \Delta^{\gamma}(z), \operatorname{Im} z>0$. (See [24] for a similar calculation using the classical theta function.) Thus, if we define $f_{\alpha, \beta}$ by

$$
f_{\alpha, \beta}(z)=\Delta^{\alpha_{0} / 12}(z) \overline{\Delta^{\bar{\beta}_{0} / 12}(z)}
$$

then we have

$$
\begin{aligned}
& f_{\alpha_{0}, \beta_{0}}(-1 / z)=e^{\pi i\left(\beta_{0}-\alpha_{0}\right) / 2} f_{\alpha_{0}, \beta_{0}}(z), \\
& f_{\alpha_{0}, \beta_{0}}(z+1)=e^{\pi i\left(\alpha_{0}-\beta_{0}\right) / 6} f_{\alpha_{0}, \beta_{0}}(z) .
\end{aligned}
$$

(This relies on the fact that the operator $g \mapsto \bar{g}$ switches and conjugates the coweights of a modular form while conjugating its multiplier system [24].) Since $f_{\alpha_{0}, \beta_{0}}$ is not identically zero (in fact is never zero), it follows that 
$v\left(\begin{array}{ll}1 & 1 \\ 0 & 1\end{array}\right)=e^{\pi i\left(\alpha_{0}-\beta_{0}\right) / 6}, v\left(\begin{array}{cc}0 & -1 \\ 1 & 0\end{array}\right)=e^{\pi i\left(\beta_{0}-\alpha_{0}\right) / 2}$ generate a multiplier system on $G_{1}$ of coweights $\alpha_{0}, \beta_{0}$.

It was previously observed in [25] that the consistency condition in coweights $\alpha_{0}, \beta_{0}$ is equivalent to the consistency condition in coweights $\alpha_{0}+2 k+w, \beta_{0}+2 l+w$ for any $k, l \in \mathbb{Z}, w \in \mathbb{C}$. Thus, $v$ just constructed is also a multiplier system on $G_{1}$ of coweights $\alpha, \beta$ ( since $\left.\alpha-\alpha_{0}, \beta-\beta_{0} \in 2 \mathbb{Z}\right)$.

To summarize: one can generate a multiplier system of arbitrary coweights $\alpha, \beta \in \mathbb{C}$ on the modular group $G_{1}$ by $v\left(\begin{array}{ll}1 & 1 \\ 0 & 1\end{array}\right)=\xi, v\left(\begin{array}{cc}0 & -1 \\ 1 & 0\end{array}\right)=\xi^{-3}$, where $\xi=\exp \{\pi i[\alpha-\beta+2(|\lfloor\operatorname{Re} \alpha\rfloor|-|\lfloor\operatorname{Re} \beta\rfloor|)] / 6\}$.

Note. To our knowledge, the oldest reference to the existence of multiplier systems of absolute value not identically one - aside from a brief mention by Petersson in 1948 [27]—is by D. James in 1973 [10].

\section{Special values of $v$}

Proposition 2. Let $v$ be a nonvanishing complex function on any real $2 \times 2$ matrix group $G$ such that $v$ satisfies the consistency condition in coweights $\alpha, \beta \in \mathbb{C}$. Then:

(i) $v(I)=1$.

(ii) If $-I \in G$, then $v(-I)= \pm \exp \{\pi i(\alpha-\beta)\}$.

(A technical condition, namely that $v(-I)=+\exp \{\pi i(\alpha-\beta)\}$, is frequently required so that nontrivial forms with that multiplier exist $[14,6$, $9,22,23]$. Since the present paper is concerned with multiplier systems themselves, we impose no such restriction.)

(iii) If $T=\left(\begin{array}{cc}0 & -1 \\ 1 & 0\end{array}\right) \in G$, then

$$
v(T)= \pm i^{\beta-\alpha+r} \quad \text { with } \quad r= \begin{cases}0 & \text { if } v(-I)=e^{i \pi(\alpha-\beta)} \\ 1 \quad \text { if } v(-I)=-e^{i \pi(\alpha-\beta)}\end{cases}
$$

Thus $v(-I)=v(T)^{-2}$.

(iv) If $S_{\lambda}=\left(\begin{array}{ll}1 & \lambda \\ 0 & 1\end{array}\right) \in G, \lambda \in \mathbb{R}$, define $\kappa$ by $v\left(S_{\lambda}\right)=\exp \{2 \pi i \kappa\}$, $0 \leq \operatorname{Re} \kappa<1$. Then $v\left(S_{n \lambda}\right)=v\left(S_{\lambda}\right)^{n}=\exp \{2 \pi i n \kappa\}$ for all $n \in \mathbb{Z}$.

Furthermore, if $S_{\lambda}, T \in G$, then:

(v) $v\left(T S_{\lambda}^{-1} T\right)=v(-I)^{-1} \exp \{2 \pi i(\alpha-\beta-\kappa)\}$.

(vi) $v\left(-T S_{\lambda} T\right)=\exp \{2 \pi i \kappa\}=v\left(S_{\lambda}\right)$.

Proof. Apply the consistency condition to the equations $I=I^{2}$, $I=(-I)^{2},-I=T^{2}$ to get (i) and to see that (i) $\Rightarrow$ (ii) $\Rightarrow$ (iii); for details, see [25], where these values were used to prove a convergence result for Poincaré series. For (iv), apply the analogous condition for $n$-fold matrix products, or use induction. For (v), (vi), use the consistency condition for 3- and 4-fold products; for details of (v) in the case $\alpha, \kappa \in \mathbb{R}, \beta=0$, see [6]. 
The coweight asymmetry $(\alpha-\beta-\kappa)$ in $(\mathrm{v})$ comes from the asymmetry in our argument convention, whence $(0 z-1)^{\alpha}(0 \bar{z}-1)^{\beta}=(-1)^{\alpha-\beta}$.

For the remainder of this section we focus on a more specific type of group. For $\lambda>0, G_{\lambda}$ is topologically discrete if and only if $\lambda>2$ or $\lambda \epsilon$ $\{1, \sqrt{2},(1+\sqrt{5}) / 2, \sqrt{3}, \ldots\}=\{2 \cos (\pi / p): p=3,4,5, \ldots\}$. The discrete Hecke groups with $\lambda<2$ are called triangle groups $[4,16]$, so named because in this case the fundamental region for $G_{\lambda}$ is a hyperbolic triangle (Figure 1).
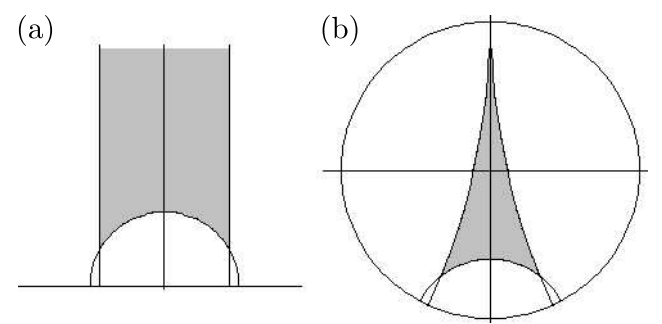

Fig. 1. (a) A fundamental region in the upper half-plane for $\left\langle\left(\begin{array}{cc}1 & \sqrt{3} \\ 0 & 1\end{array}\right),\left(\begin{array}{cc}0 & -1 \\ 1 & 0\end{array}\right)\right\rangle$, which is the case $p=6$. Here $\mathcal{R}=\{z \in \mathbb{C}:|z|>1, \operatorname{Re} z<\sqrt{3} / 2, \operatorname{Im} z>0\}$.

(b) The same hyperbolic triangle represented in the Poincaré disc, via the conformal map $z \mapsto i(z-4 i) /(z+4 i)$.

Our next two results connect the four parameters associated with multiplier systems on triangle groups. The first is a congruence.

Proposition 3. Let $v$ be a multiplier system of coweights $\alpha, \beta \in \mathbb{C}$ on a Hecke triangle group $G_{\lambda}, \lambda=2 \cos (\pi / p), p \in\{3,4,5, \ldots\}$. Define $\kappa$ by $v\left(S_{\lambda}\right)=\exp (2 \pi i \kappa), 0 \leq \operatorname{Re} \kappa<1$. Then

$$
(p-2)(\alpha-\beta)-4 p \kappa \in \mathbb{Z},
$$

and in particular

$$
\begin{aligned}
(p-2)(\alpha-\beta)-4 p \kappa & \equiv 2 \pm p \text { or } p \pm p(\bmod 4) \\
& \equiv \frac{1}{2}\{(p+2) \pm(p-2) \pm 2 p\}(\bmod 4) .
\end{aligned}
$$

Proof. Apply the consistency condition $p-1$ times to the group relation $\left(S_{\lambda} T\right)^{p}=-I$, take $z=e^{\pi i / p}$ and use Proposition 2(iii). (The preceding steps mirror a calculation for real weight and bounded $v$ in [6].) Modulo 4, then,

$$
(p-2)(\alpha-\beta)-4 p \kappa \equiv \begin{cases}2+p & \text { if } v(T)=i e^{\pi i(\beta-\alpha) / 2} \\ 2-p & \text { if } v(T)=-i e^{\pi i(\beta-\alpha) / 2} \\ 2 p & \text { if } v(T)=-e^{\pi i(\beta-\alpha) / 2} \\ 0 & \text { else. }\end{cases}
$$

This implies the conclusions of Proposition 3. 
A similar group relation holds for certain of the nondiscrete Hecke groups as well [7], and so these results can be extended slightly if desired.

Corollary 1. Let $G_{\lambda}$ be a Hecke triangle group, and $\kappa, \alpha, \beta$ be defined as before. Thus $v\left(S_{\lambda}\right)=e^{2 \pi i \kappa}$ with $0 \leq \operatorname{Re} \kappa<1$, or equivalently, $\kappa=$ $1 / 2+(2 \pi i)^{-1} \log \left[-v\left(S_{\lambda}\right)\right]$. Then $\kappa \in \mathbb{R} \Leftrightarrow \alpha-\beta \in \mathbb{R}$.

Proof. By Proposition $3,(p-2) \operatorname{Im}(\alpha-\beta)=4 p \operatorname{Im} \kappa$; the corollary follows.

Corollary 2. For fixed complex coweights, an upper bound on the number of multiplier systems on a triangle group is given by $n(\lambda, \alpha, \beta)=$ $4 \pi / \cos ^{-1}(\lambda / 2)$.

Proof. By the proof of Proposition 3,

$$
(p-2)(\alpha-\beta)-4 p \kappa= \begin{cases}4 j+2+p & \text { if } v(T)=i e^{\pi i(\beta-\alpha) / 2} \\ 4 j+2-p & \text { if } v(T)=-i e^{\pi i(\beta-\alpha) / 2} \\ 4 j+2 p & \text { if } v(T)=-e^{\pi i(\beta-\alpha) / 2} \\ 4 j & \text { else. }\end{cases}
$$

Because $0 \leq \operatorname{Re} \kappa<1, j$ lies in a left-open interval of length $p$. Since $p \in \mathbb{Z}^{+}$, in each of the four cases above there are at most $p$ possible values for $j$, and thus for $\kappa$. This gives us a bound of $4 p$. (For instance, when $p=3, \alpha=2+3 i$ and $\beta=i$, the four cases correspond to $4 j$ lying in

$$
\begin{aligned}
& ((p-2) \operatorname{Re}(\alpha-\beta)-5 p-2,(p-2) \operatorname{Re}(\alpha-\beta)-p-2]=(-15,-3], \\
& ((p-2) \operatorname{Re}(\alpha-\beta)-3 p-2,(p-2) \operatorname{Re}(\alpha-\beta)+p-2]=(-9,3], \\
& ((p-2) \operatorname{Re}(\alpha-\beta)-6 p,(p-2) \operatorname{Re}(\alpha-\beta)-2 p]=(-16,-4], \\
& ((p-2) \operatorname{Re}(\alpha-\beta)-4 p,(p-2) \operatorname{Re}(\alpha-\beta)]=(-10,2],
\end{aligned}
$$

respectively, so there are at most 12 multipliers on $G_{1}$ of coweights $2+3 i, i$. This is consistent with the results of Rademacher and Zuckerman on the modular group for real weight [31].)

Therefore a bound is given by $n(\lambda, \alpha, \beta)=4 p=4 \pi / \cos ^{-1}(\lambda / 2)$. (Since $0<\pi / p<\pi / 2$, we have $\lambda=2 \cos (\pi / p) \Leftrightarrow p=\pi / \cos ^{-1}(\lambda / 2)$.) Note that this bound is independent of the coweights.

Having calculated the possible values $v$ can take on the generators $T$ and $S_{\lambda}$ of $G_{\lambda}$ (by finding $v(T)$ in Proposition 2(iii), $\operatorname{Im} \kappa$ in the proof of Corollary 1, and Re $\kappa$ in the proof of Corollary 2), we are well on the way to a characterization of the multiplier systems on Hecke triangle groups. What is still needed is a converse of some sort, establishing which of these possible multipliers actually do satisfy the consistency condition for all choices of $M_{1}, M_{2}$. 
Now for an application of the foregoing results. In [25] it was proved that under the restriction $\left|v\left(S_{1}\right)\right|=1$, Poincaré series on the modular group do not converge nicely when $\alpha-\beta \notin \mathbb{R}$. That restriction can now be removed, and a more general result proved, with the aid of Proposition 3. This is the essence of our next corollary.

Corollary 3. If $\alpha-\beta \notin \mathbb{R}$, then Petersson's Poincaré series on the modular group is not absolutely convergent, for any multiplier system. Thus for complex weight in general it is not possible to build modular forms in the usual way, even allowing for a broader definition of multiplier than usual. (While modular forms of nonreal coweight difference do exist, they cannot be built from a basis of Poincaré series.)

Proof. [25] gives a sufficient condition for Poincaré series on the modular group to fail to converge absolutely, namely that $\operatorname{Im}(\alpha-\beta)+(2 \pi)^{-1} \log \left|v\left(S_{2}\right)\right|$ $\neq 0$. (Omitted parentheses have been restored here.) This condition allows one to obtain an expression for $\left|v\left(\left(S_{2} T\right)^{n}\right)\right|$ that causes the absolute subseries on the cyclic subgroup $\left\langle S_{2} T\right\rangle$ to diverge.

On the modular group we have $\lambda=1, p=3$. Also, $v\left(S_{2}\right)=v\left(S_{1}^{2}\right)=$ $v\left(S_{1}\right)^{2}=e^{4 \pi i \kappa}$, and so

$$
\begin{aligned}
\operatorname{Im}(\alpha-\beta)+(2 \pi)^{-1} & \log \left|v\left(S_{2}\right)\right| \\
& =\operatorname{Im}(\alpha-\beta)+(2 \pi)^{-1}(-4 \pi \operatorname{Im} \kappa) \\
& =\operatorname{Im}(\alpha-\beta)-2 \operatorname{Im} \kappa \\
& =(p-2) \operatorname{Im}(\alpha-\beta)-2 \operatorname{Im} \kappa \quad \text { because } p=3 \\
& =4 p \operatorname{Im} \kappa-2 \operatorname{Im} \kappa \quad \text { by the proof of Corollary } 1 \\
& =10 \operatorname{Im} \kappa \neq 0 \quad \text { by Corollary } 1, \text { since } \alpha-\beta \notin \mathbb{R} .
\end{aligned}
$$

Thus the aforementioned sufficient condition is met, and the Poincaré series does not converge absolutely. (Therefore the usual method of forming a basis for the space of modular forms will not work for general multipliers.)

It bears mention that this divergence problem is not remedied by a Hecke convergence factor. For, a Poincaré series of coweights $\alpha, \beta$ with convergence factor $|c z+d|^{-s}$ inserted can simply be reinterpreted as a form of coweights $\alpha+s / 2, \beta+s / 2$; the coweight difference is unchanged, and Corollary 3 still applies.

4. The theta group. What of the remaining discrete Hecke groups? That is, can anything be said about $\kappa$ when $\lambda \geq 2$ ? Lacking a group relation of the type $\left(S_{\lambda} T\right)^{p}=-I$, we are without recourse to the arguments used in the proof of Proposition 3. (For $\lambda \geq 2$, the only group relation is $T^{2}=-I$, which we have already fully utilized in Proposition 2(iii).) 
In particular, consider the limiting case: the theta group, $G_{2}$. Since $2=$ $\lim _{p \rightarrow \infty} 2 \cos (\pi / p)$, we might hope to glean some information about at least this one specific case from Proposition 3 and/or Corollary 1. Intuitively, since by Proposition 3 it is true that $(1-2 / p)(\alpha-\beta)-4 \kappa \in \mathbb{R}$ for $p=$ $3,4,5, \ldots$, in the limit one might expect likewise that $\operatorname{Im} \kappa=\operatorname{Im}(\alpha-\beta) / 4$ for multiplier systems on $G_{2}$. However, in [25] we find that for any $\alpha, \beta \in \mathbb{C}$ there exists a multiplier system on $G_{2}$ with $\kappa=0$. Thus no result analogous to Proposition 3 and/or Corollary 1 extends to $\lambda=2$; the multiple-valued function $\Upsilon: \lambda \mapsto \kappa(\lambda)$ is not continuous.

\section{References}

[1] A. N. Andrianov, Symmetries of harmonic theta functions of integer-valued quadratic forms, Uspekhi Mat. Nauk 50 (1995), no. 4, 3-44 (in Russian); English transl.: Russian Math. Surveys 50 (1995), 661-700.

[2] T. M. Apostol, Modular Functions and Dirichlet Series in Number Theory, 2nd ed., Springer, New York, Berlin, 1990.

[3] W. L. Baily, Jr., Introductory Lectures on Automorphic Forms, Princeton Univ. Press, Princeton, 1973.

[4] A. F. Beardon, The Geometry of Discrete Groups, Springer, New York, Berlin, 1995.

[5] T. Dern, Multiplikatorsysteme und Charaktere Hermitescher Modulgruppen, Monatsh. Math. 126 (1998), 109-116.

[6] A. Hassen, Log-polynomial period functions for Hecke groups, Ramanujan J. 3 (1999), 119-151.

[7] -, Log-polynomial period functions for nondiscrete Hecke groups, Proc. Amer. Math. Soc. 128 (2000), 387-396.

[8] E. Hecke, Über die Bestimmung Dirichletscher Reihen durch ihre Funktionalgleichung, Math. Ann. 112 (1936), 664-699.

[9] H. Iwaniec, Topics in Classical Automorphic Forms, Grad. Stud. in Math. 17, Amer. Math. Soc., Providence, 1997.

[10] D. A. James, Functions automorphic on large domains, Trans. Amer. Math. Soc. 181 (1973), 385-400.

[11] M. I. Knopp, Determination of certain roots of unity in the theory of automorphic forms of dimension zero, Duke Math. J. 27 (1960), 497-506.

[12] - Construction of automorphic forms on H-groups and supplementary Fourier series, Trans. Amer. Math. Soc. 103 (1962), 168-188.

[13] —, Polynomial automorphic forms and nondiscontinuous groups, ibid. 123 (1966), $506-520$.

[14] —, Modular Functions in Analytic Number Theory, 2nd ed., Chelsea, New York, 1993.

[15] M. I. Knopp and G. Mason, Generalized modular forms, J. Number Theory, to appear.

[16] M. I. Knopp and M. Sheingorn, On Dirichlet series and Hecke triangle groups of infinite volume, Acta Arith. 76 (1996), 227-244.

[17] A. Krieg, The Maass-space for the non-trivial multiplier system over the Hurwitz quaternions, Arch. Math. (Basel) 70 (1998), 211-218. 
[18] A. Krieg and S. Walcher, Multiplier systems for the modular group on the 27dimensional exceptional domain, Comm. Algebra 26 (1998), 1409-1417.

[19] J. Lehner, Discontinuous Groups and Automorphic Functions, Math. Surveys Monogr. 8, Amer. Math. Soc., Providence, 1964.

[20] H. Maass, Lectures on Modular Functions of One Complex Variable, Lectures on Math. 29, Tata Institute of Fundamental Research, Bombay, 1964.

[21] D. Niebur, Construction of automorphic forms and integrals, Trans. Amer. Math. Soc. 191 (1974), 373-385.

[22] L. A. Parson, Dedekind sums and noncongruence subgroups of the Hecke groups $G(\sqrt{2})$ and $G(\sqrt{3})$, Proc. Amer. Math. Soc. 57 (1976), 194-196.

[23] - Generalized Kloosterman sums and the Fourier coefficients of cusp forms, Trans. Amer. Math. Soc. 217 (1976), 329-350.

[24] P. C. Pasles, Nonanalytic automorphic integrals on the Hecke groups, Acta Arith. 92 (1999), 155-171.

[25] - Convergence of Poincaré series with two complex coweights, in: Contemp. Math. 251, Amer. Math. Soc., Providence, 2000, 453-461.

[26] - , A Hecke correspondence theorem for nonanalytic automorphic integrals, J. Number Theory 83 (2000), 256-281.

[27] H. Petersson, Automorphe Formen als metrische Invarianten, I, II, Math. Nachr. 1 (1948), 158-212 and 218-257.

[28] —, Über Modulfunktionen und Partitionenprobleme, Abh. Deutsch. Akad. Wiss. Berlin. Kl. Math. Allg. Nat. 1954, no. 2, 59 pp.

[29] N. Proskurin, Cubic Metaplectic Forms and Theta Functions, Lecture Notes in Math. 1677, Springer, Berlin, 1998.

[30] H. Rademacher, Zur Theorie der Modulfunktionen, J. Reine Angew. Math. 167 (1932), 312-336.

[31] H. Rademacher and H. S. Zuckerman, On the Fourier coefficients of certain modular forms of positive dimension, Ann. of Math. (2) 39 (1938), 433-462.

[32] B. Schoeneberg, Elliptic Modular Functions: An Introduction, Springer, New York, Heidelberg, 1974.

[33] J. R. Smart, Parametrization of automorphic forms for the Hecke groups $G(\sqrt{2})$ and $G(\sqrt{3})$, Duke Math. J. 31 (1964), 395-403.

[34] X. L. Wang, Poincaré series with arbitrary positive real weights I: The nonanalytic case, Acta Math. Sinica 37 (1994), 767-782 (in Chinese).

Department of Mathematical Sciences

Villanova University

800 Lancaster Avenue

Villanova, PA 19085, U.S.A.

E-mail: paul.pasles@villanova.edu

URL: http://www.pasles.org

Received on 25.10.2001

and in revised form on 29.10.2002 